\title{
Sleep and wakefulness in the cuneate nucleus: a computational study
}

\author{
Eduardo Sánchez ${ }^{1}$, Senén Barro ${ }^{1}$, Jorge Mariño ${ }^{3}$, and Antonio Canedo ${ }^{2}$ \\ 1 Grupo de Sistemas Intelixentes (GSI) \\ Departamento de Electrónica e Computación, Facultade de Físicas, \\ Universidade de Santiago de Compostela, \\ 15782 Santiago de Compostela, Spain \\ \{eduardos, elsenen\}@usc.es \\ http://www-gsi.usc.es/index.html \\ 2 Departamento de Fisioloxía, Facultade de Medicina, \\ Universidade de Santiago de Compostela, \\ 15782 Santiago de Compostela, Spain \\ fsancala@usc.es \\ 3 Departamento de Medicina, Escola Universitaria de Fisioterapia, \\ Universidade da Coruña, \\ 15006 A Corua, Spain \\ xurxo@mit.edu
}

\begin{abstract}
We present a computational study about the influence of the sensorimotor cortex on the processing of the cuneate nucleus during sleep as well as wakefulness. Realistic computational models were developed supported by experimental data obtained from intact-brain preparations in cat. Furthermore, a physiologically plausible circuit is proposed and predictions under both different cortical stimulation and synaptic configurations are suggested. The computer simulations show that the CN circuitry (1) under sleep conditions can block the transmission of afferent sensory information, and (2) under awaking conditions can perform operations such as filtering and facilitation.
\end{abstract}

\section{Introduction}

The cuneate nucleus $(\mathrm{CN})$ is located within the brain stem in the most rostral area of the spinal chord, between the Obex and $4 \mathrm{~mm}$ caudal to it. This zone is composed of a core or central region, and a shell or peripheral region. The core is basically made up of clusters of cuneothalamic neurones, also referred to as projection or relay neurones, which are surrounded by interneurons, also known as local neurons or non-thalamic projection neurones. The shell, comprising the ventral and marginal regions, is made up of interneurons and other projecting neurons. Cuneothalamic cells project their axons to the VPL nucleus whereas interneurons exert their influence over the cuneothalamic neurons as well as on other interneurons.

The CN receives four main types of afferent fibres: primary afferent fibres from the cutaneous and deep receptors, fibres coming from the brainstem, direct 
corticocuneate fibres, and collaterals of the corticospinal tract originated in the cerebral cortex. The cutaneous afferent fibres are confined basically to the core or central region of the medial cuneate and mostly establish axodendritic synapses [4]. The descending direct and collaterals fibres can be grouped based on their origin within the sensorimotor cortex (SMC): those originating in Brodmann's areas 4 (motor cortex) and 3a (proprioceptive somatosensory cortex) contact the shell [3]; those from area $3 \mathrm{~b}$ (cutaneous somatosensory cortex) project directly to the core; and those from areas 1 and 2 (cutaneous somatosensory cortex) project directly into the shell as well as into the core but within zones surrounding the cluster of cuneothalamic cells.

With regard to the effects of the descending fibres, several studies [7] found that the sensorimotor cortex either excites, blocks or does not affect the cell activity. Gordon and Jukes [5] studied the target cells of the descending fibres and found that cortical stimulation inhibits neurons in the medial zone. This inhibition, classically explained by means of a presynaptic mechanism, is still an open issue after recent experiments reporting evidences of postsynaptic rather than presynaptic inhibition [1]. On the other hand, it has been demonstrated that the sensorimotor cortex could directly excite cuneothalamic cells of the CN $[2]$.

Our interest is focused on the role of the corticocuneate circuitry in sleep and wakefulness. About wakefulness, it was proposed that the SMC could control the information flow through the $\mathrm{CN}$ in two complementary ways [11]: (1) facilitating the transmission of signals originated in those peripheral areas which either processes a high intensity stimulus or they are inside the cortical attentional window; and (2) filtering irrelevant information. About sleep, recent findings show that cortical descending fibres play an important role by imposing oscillatory rhythms in the CN [9]. These rhythms, intensively studied in the thalamus [10], are supposed to be in charge of blocking the ascending transmission at subcortical level.

In this paper we propose a plausible corticocuneate circuitry and study how it might work in both sleep and awaking conditions. The presentation is organized as follows: (1) a brief description of experimental and computational methods, (2) the introduction of the corticocuneate circuitry, (3) computer experiments under sleep conditions, (4) computer experiments under awaking conditions, and (4) a final discussion.

\section{Methods}

\section{$2.1 \quad$ Experimental}

All experiments conformed to Spanish guidelines (BOE 67/1998) and European Communities council directive (86/609/EEC). Moreover, all efforts were made to minimize the animals used. Data were obtained from cats $(2.3-4.5 \mathrm{~kg})$, which were anesthetized ( $\alpha$-cloralosa, $60 \mathrm{mg} / \mathrm{Kg}$ ), i.p.), paralyzed (Pavulon, $1 \mathrm{mg} / \mathrm{kg} / \mathrm{h}$, i.v) and placed under artificial respiration. A set of six bipolar stimulating electrodes 
was mounted in a tower and lowered to 1-1.5 $\mathrm{mm}$ deep in the pericruciate cortex to stimulate corticocuneate neurons. The cortical electrodes were disposed so that primary motor and primary somatosensory cortices could be stimulated. A bipolar concentric electrode and sharp electrodes were used to record the electrocorticogram (EcoG) and to record intracellularly from cuneate neurons, respectively. All cuneolemniscal cells satisfied the collision test with spontaneous action potentials.

\subsection{Computational}

Neuron level The multi-compartmental approach was used to model the neurons. A detailed description of the compartments and the ionic currents used for each neuron can be found in Sánchez et al. [13].

Membrane level All ionic currents are described by following the HodgkinHuxley model. The mathematical description of all this currents can be found in Sánchez et al. [14].

Synaptic level To describe the conductance associated with the synaptic current we have used the alpha function [8]. The synaptic current is determined by $I_{\text {syn }}(t)=g_{\text {syn }}(t)\left(V-V_{\text {syn }}\right)$, where $V_{\text {syn }}$ denotes the resting potential of the postsynaptic membrane. In our model we introduce both excitatory and inhibitory connections. We assume the later being glutamatergic and the former being gabaergic. The excitatory connections are modeled by setting $V_{\text {syn }}$ to zero and the inhibitory connections to a negative value.

Simulation tools We have used Neuron as it provides an optimised integration method developed by Hines [6]. The machine used for the simulations was a PC with a $600 \mathrm{Mhz}$ AMD Athlon processor.

\section{Results}

\subsection{Corticocuneate circuitry}

Experiments were performed in intact-brain preparations in anaesthetized cats. Simultaneous recordings in the sensorimotor cortex (SMC) and the cuneate nucleus $(\mathrm{CN})$ were obtained while the SMC was stimulated with 1-10 Hz electrical pulses, whereas intracellular recordings were prepared to study the effects of SMC stimulation over CN cells. Figure 1(A) shows selected recordings providing experimental evidence of excitation and inhibition of $\mathrm{CN}$ cells. According to these data, a circuit is proposed (figure 1(B)) and a computational model is developed in order to explain the observed effects. For the CN neurons we have used realistic computational models $[12,14]$ that were constructed based on data from current injection experiments and validated against neuronal activity recorded in vivo in anaesthetized cats. 


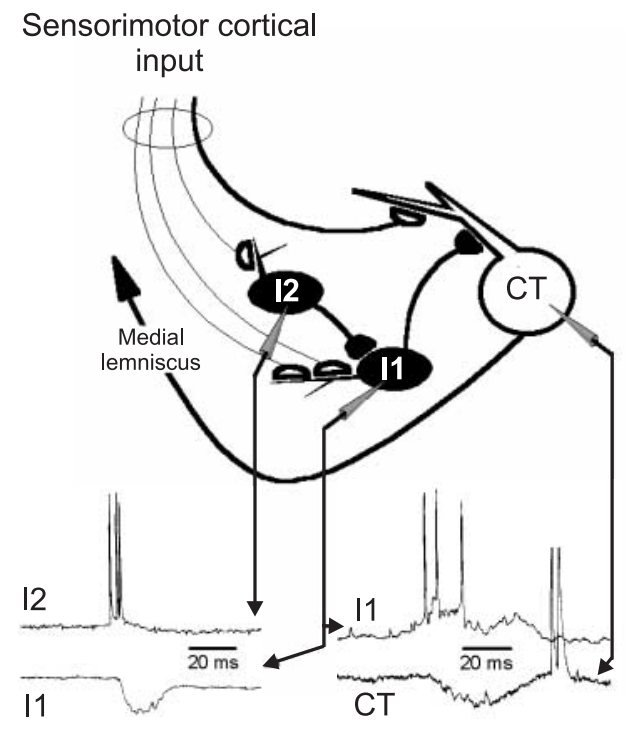

A

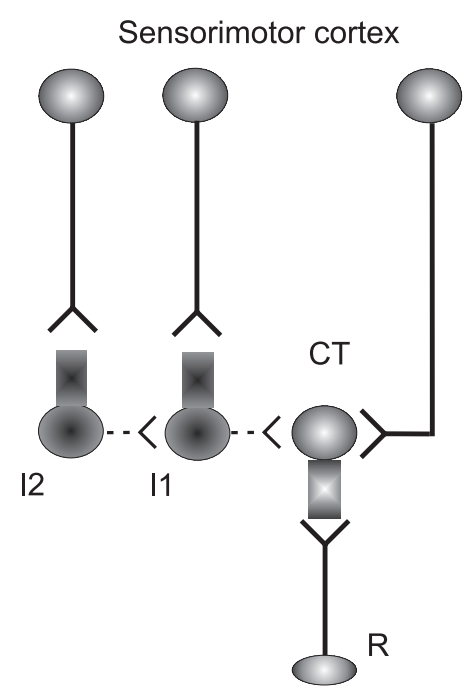

B

Fig. 1. The corticocuneate circuitry. (A) Intracellular recordings show excitation and inhibition effects over both cuneothalamic (CT) neurons and interneurons (I1 and I2). (B) The circuitry considered for simulation: cortical excitation and inhibition over interneurons, cortical inhibition over cuneothalamic neurons, and cortical excitation over cuneothalamic neurons.

\subsection{Sleep state}

The synapses of the computational model were adjusted by comparing simulated outcomes with experimental neuronal activity recorded in anaesthetized cats. The model is therefore tuned to sleep state conditions, and ready to test whether the corticocuneate circuitry can accomplish the hypothesized blockade of sensory information. Figure 2 shows a cuneothalamic neuron (CT) being inhibited after cortical stimulation. Initially, the inhibition hyperpolarizes the neuron and activates $I_{h}$ and $I_{T}$ currents, which leads to a burst of spikes and the beginning of a spindle-like rhythm. Next, we activate a sensory receptor to generate afferent signals. At the hyperpolarization subcycle, the firing threshold is so high that sensory signals can only drive a small membrane depolarisation. At the depolarised subcycle, the intrinsic-generated spikes completely mask the processing of afferent signals. Therefore, the sensory information do not alter the oscillatory pattern, and the signal is completely blocked.

But, how does the complete circuitry work? To analyze this point we have set a simulation with the whole circuitry depicted in figure 1 . We have stimulated the cortical neurons that elicit inhibition (CI) and excitation (CE) over 


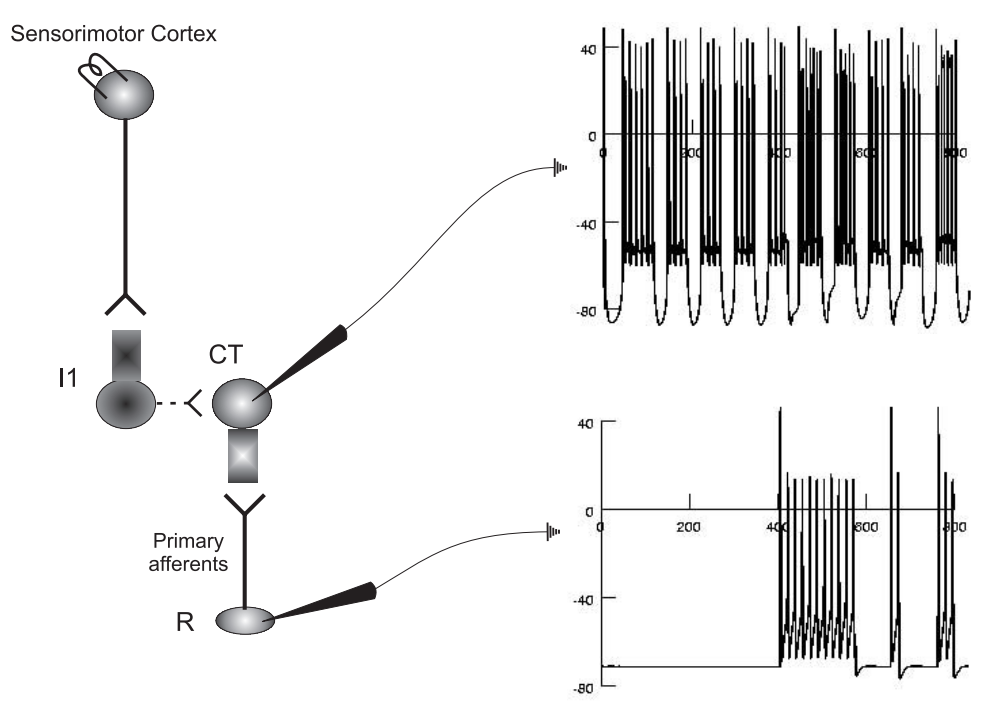

Fig. 2. Oscillatory activity and blockade of afferent information. The inhibition originated from the sensorimotor cortex elicits an oscillatory bursting response over the CT neuron (upper right). From 400 to $800 \mathrm{~ms}$, cutaneous stimulation is generated with different temporal durations (lower right), but the oscillatory activity blocks the transmission of this information.

CT neurons (figure 3). We have also assumed an interconnection between $\mathrm{CE}$ and cortical neurons that inhibit interneurons (CD) [2]. The simulations indicate that the duration of both depolarized and hyperpolarized subcycles periods is longer if comparing them with the spontaneous activity in figure 3 . The hyperpolarized subcycle duration is explained because we have simulated a low rhythm in the SMC, therefore the CT neurons are inhibited during a longer period of time. The depolarized subcycle is explained because of the excitatory cortical descending fibres providing excitation. In fact, we have found that this is the unique mechanism to accomplish an activity pattern similar to that observed in vivo [9]. So, we predict that the excitatory connections play a fundamental role in the shaping of the slow rhythms at the $\mathrm{CN}$ determining the duration of the spike subcycle.

\subsection{Awaking state}

And now we question the functioning of the corticocuneate circuitry in wakefulness. Considering the Mountcastle approach, the SMC would influence the CN to perform two main tasks: filtering of irrelevant or unwanted information, and facilitation of relevant stimuli. To model this behaviour we first need to consider what it means to be at wakefulness. In other words, what should we change in our model to satisfy the awaking conditions?. In the cuneate nucleus, brain stem 

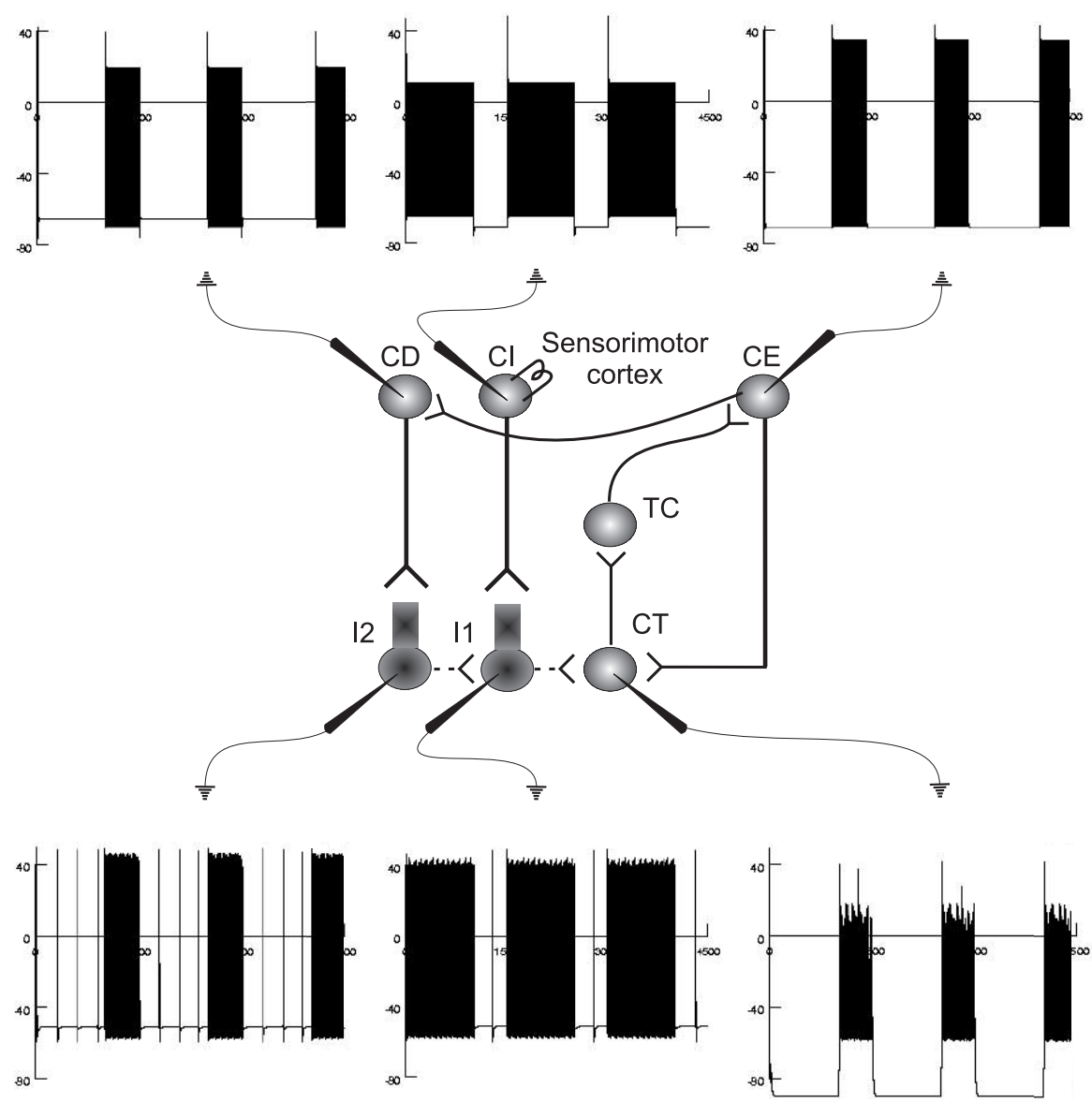

Fig. 3. Slow rhythms and the participation of excitatory synapses on their activity pattern. A sensorimotor cortical neuron is stimulated to induce a slow rhythm $(<$ $1 \mathrm{~Hz}$ ) that spreads throughout the entire corticocuneate circuitry. As a result, the CT neuron shows an oscillatory activity very similar to that observed in vivo. The hyperpolarized subcycle is determined by the duration of inhibition carried out by I1. The depolarised bursting subcycle is determined by the duration of the cortical excitatory effects maintained through the CT-TC loop.

or cortical afferents could induce similar effects to those observed in the thalamus [15] and be in charge of inducing the transition from sleep to wakefulness. We have simulated these effects by changing $V_{\text {syn }}$ from $-90 \mathrm{mV}$ to $-75 \mathrm{mV}$. In this way, we avoid the activation of $I_{h}$ and $I_{T}$ currents.

In figure 4 we show a circuitry that integrates the three descending pathways in charge of inhibition, excitation and desinhibition of cuneothalamic neurons. We have also connected, as proposed by Canedo et al. [2], the cortical neuron 


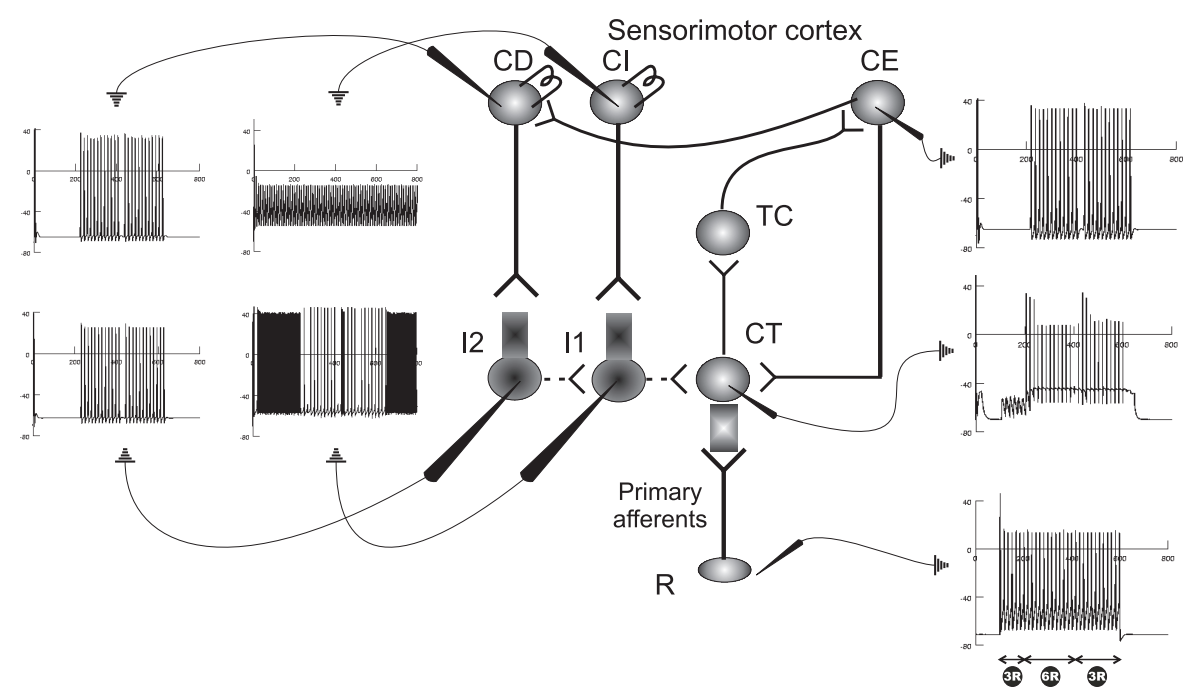

Fig. 4. Filtering and facilitation. Cutaneous stimulation of 3 receptors (R) cannot overcome the filtering originated from the sensorimotor cortex. We need 6 receptors (from 200 to $400 \mathrm{~ms}$ ) to completely transmit the information. When we set back the number of activated receptors to 3 , both cortical excitation and desinhibition facilitates the transmission of cutaneous information.

in charge of cuneate excitation with the other one in charge of cuneate desinhibition. The synapse between interneurons was set with $g_{s y n}^{\max }=0.1$ and $\tau=4$. Under activation of 3 receptors $(0.005 \mathrm{nA})$ during $100-200 \mathrm{~ms}$, there is no response in the cuneothalamic neuron because of the cortical inhibition imposed by CI. After increasing the activation to 6 receptors in the interval 200-400 $\mathrm{ms}$, the cuneothalamic neuron overcomes the cortical filtering generating action potentials, and thus activating, through the thalamocortical (TC) neuron, the excitatory cortical neuron. This one activates the desinhibitory cortical neuron, which, in turn, activates the corresponding cuneate interneuron. The final effect is a partial inhibition of the interneuron I2, which decreases substantially its activity. The most salient aspect can be found in the interval from 400 to $600 \mathrm{~ms}$, in which the activated receptors was reduced to 3 . The cortical action facilitates the transmission of cutaneous information by maintaining a sustained depolarisation over the cuneothalamic neuron. The simulation finalizes, after 600 $\mathrm{ms}$, when the stimulation is removed and the cuneothalamic neuron ceases its activity.

\section{Discusion}

The main contribution of this work is to show how the same circuitry can perform completely different tasks during sleep and wakefulness. These states impose gen- 
eral conditions that determine not only the activity of the cuneate nucleus, but also the activity of the overall brain. We hope that the study presented here will encourage further research concerning how the circuits studied in anaesthetized or sleep conditions can perform tasks during wakefulness.

\section{Acknowledgments}

We would like to thank to Laboratorios de Neurociencia y Computación neuronal (LANCON), the environment in which this work has been developed, and the support from the Xunta de Galicia through grant PGIDIT-02TIC20601PR.

\section{References}

1. Canedo, A.: Primary motor cortex influences on the descending and ascending systems. Progress in Neurobiology. Vol. 51 (1997) 287-335

2. Canedo A., Aguilar J., Mariño J.: Lemniscal recurrent and transcortical influences on cuneate neurons. Neuroscience. Vol. 972 (2000) 317-334

3. Cheema SS, Fyffe RE, Light A, Rustioni A: Arborizations of single corticofugal axons in the feline cuneate nucleus stained by iontophoretic injection of horseradish peroxidase. Brain research Vol. 290. (1984) 158-164

4. Fyffe Robert E., Cheema Surindar S., Rustioni A.: Intracelular Staining Study of the Feline Cuneate Nucleus. I. Terminal Patterns of Primary Afferent Fibers. Journal of Neurophysiology. Vol. 56. 5 (1986) 1268-1283

5. Gordon G, Jukes MGM: Descending influences on the exteroceptive organizations of the cats gracile nucleus. J. Physiol. (London) Vol. 173 (1964) 291-319

6. Hines M.: A program for simulation of nerve equations with branching geometries. International Journal of Biomedical Computation. Vol. 24 (1989) 55-68

7. Jabbur SJ, Towe AL: Cortical excitation of neurons in dorsal column nuclei of cat. J. Neurophysiology Vol. 24 499-509

8. Jack J. J. B., Redman S. J.: The propagation of transient potentials in some linear cable structures. J. of Physiology (London). Vol. 215 (1971) 283-320

9. Mariño J, Canedo A, Aguilar J: Sensorimotor cortical influences on cuneate nucleus rhythmic activity in the anesthetized cat. Neuroscience Vol. 953 (2000) 657-673

10. McCormick DA, Bal T: Sleep and arousal: Thalamocortical mechanisms Annual Review Neuroscience Vol. 20 (1997) 185-215

11. Mountcastle VB, Darian-Smith I: Neural mechanisms in somesthesia In Medical Physiology. Mosby, St. Louis. Mountcastle VB (Ed). (1968) 1372-1423

12. Sánchez E., Barro, S., Canedo, A., Martìnez, L., Mariño, J.: A computational model of cuneate nucleus interneurons. Eur. J. Neurosci. Vol. 10. 10 (1998) 402

13. Sánchez E, Barro S, Mariño J, Canedo A: A realistic computational model of the local circuitry of the cuneate nucleus In Lecture Notes in Computer Science. Volume I. Springer Verlag. Mira J. and Prieto A. (Eds). (2001) 21-29.

14. Sánchez E, Barro S, Mariño J, Canedo A: A computational model of cuneothalalamic projection neurones. Network: Computation in neural systems. Vol. 14. (2003) 211-231

15. Steriade M, McCormick DA, Sejnowski TJ: Thalamocortical oscillations in the sleeping and aroused brain. Science Vol. 262. (1993) 679-685 